\title{
RESEARCH
}

Open Access

\section{Feasibility and impact of using an automated user interface for proton spectroscopy in multiparametric MRI of the prostate}

Mohamed M. H. Abd Ellah ${ }^{1,2,3^{*}}$, Christian Kremser ${ }^{1}$, Werner Judmaier ${ }^{1}$, Berthold Kiefer ${ }^{4}$, Martin Requardt ${ }^{4}$, Radhouene Neji ${ }^{5}$, Hosam M. Kamel ${ }^{2}$ and Daniel Junker ${ }^{1}$

\begin{abstract}
Background: Multiparametric MRI including MR spectroscopy has recently gained much acceptance being a noninvasive imaging modality for the evaluation of prostate cancer. This study aimed to evaluate the reliability and effectiveness of an assisted workflow for guided and automated multiparametric MRI (mpMRI) of the prostate including MR spectroscopy (MRS) on a 3 T scanner.

Fifty-six consecutive patients (age range 44-73 years, mean 63 years) with suspected prostate cancer underwent mpMRI using a surface coil in a 3 T MR scanner. Thirty-eight patients were examined using a newly developed computer-assisted workflow and a control group of 18 randomly selected patients with the conventional technique. Overall examination time, spectral quality, saturation pulse positioning, and the outcome of automatic prostate segmentation were compared. A grading of 1 to 5 was used, where 1 represents the highest and 5 the lowest quality of the respective results.
\end{abstract}

Results: Both the conventional technique and the computer-assisted workflow provided good spectral quality. No significant differences were found regarding saturation pulse positioning and spectral quality $(P>0.05)$. However, a significant difference regarding overall examination time was found, 40.78 min versus 32.6 min for conventional and computer-assisted workflow respectively $(P<0.001)$. The automated scanning technique also showed satisfactory results for automated prostate segmentation.

Conclusions: The computer-assisted workflow achieved good spectral quality while reducing overall examination time. This time saving effect may allow the inclusion of MRS in clinical routine.

Keywords: Prostate neoplasms, Magnetic resonance imaging, Functional magnetic resonance imaging, MR spectroscopy, Prostate cancer

\footnotetext{
*Correspondence: dr_m_hamdy2006@hotmail.com;

mohamed.hamdy.abdellah@gmail.com

'Department of Radiology, Medical University Innsbruck, Anich St. 35, 6020

Innsbruck, Austria

2Department of Diagnostic Radiology, South Egypt Cancer Institute, Assiut

University, Assiut, Egypt

Full list of author information is available at the end of the article
}

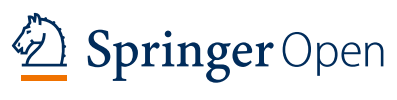

(c) The Author(s). 2020 Open Access This article is licensed under a Creative Commons Attribution 4.0 International License which permits use, sharing, adaptation, distribution and reproduction in any medium or format, as long as you give appropriate credit to the original author(s) and the source, provide a link to the Creative Commons licence, and indicate if changes were made. The images or other third party material in this article are included in the article's Creative Commons licence, unless indicated otherwise in a credit line to the material. If material is not included in the article's Creative Commons licence and your intended use is not permitted by statutory regulation or exceeds the permitted use, you will need to obtain permission directly from the copyright holder. To view a copy of this licence, visit http://creativecommons.org/licenses/by/4.0/. 


\section{Background}

In the field of male genitourinary diseases, prostate cancer is one of the main challenges for urologists, oncologists, and radiologists. It is the third leading cause of overall cancer-related death in men in both Europe and USA and only outnumbered by lung and colorectal cancer deaths. The incidence of new cases has been reported to be about 218,890 cases in 2007 in the USA and about 30 per 100,000 men in Europe [1-3]. It is even more progressing with the new records in 2018 reported 1.3 million newly diagnosed cases and 359,000 cancer-related deaths [4].

Magnetic resonance imaging (MRI) has recently been accepted as a non-invasive primary imaging modality of choice for the evaluation of prostate cancer. It proved efficiency in detection, localization, and staging of prostate cancer. Apart from standard morphological imaging, additional sequences yielding supplemental functional information are used in multiparametric MRI (mpMRI) [5-10].

In addition, magnetic resonance spectroscopy (MRS) has been used to gain biochemical information further improving tumor detection rate. In fact, MRS was one of the first well-investigated functional constituents of mpMRI $[11,12]$.

Metabolite ratios (cho+cr/cit and cho+cr) also proved in a recently published study [12] to have the best discriminating ability for significant Gleason score lesions $(\geq 5)$.

Despite its proven usefulness in prostate cancer detection, localization, as well as guiding and monitoring therapy, MRS is still confronted with some limitations, foremost the lengthy acquisition time. The known interobserver variability in post-processing and evaluation of the results has to be addressed by specialized training and experience and diminishes through expertise with increasing use of the method [13, 14]. For MRI technicians, one of the main challenges in performing MRS of the prostate is the correct positioning of saturation pulses encompassing the whole gland. This is needed for outer volume suppression (OVS) thus avoiding spectra contamination from surrounding lipids and from the water signal in the bladder. The planning of the prostate MRS exam also requires the correct positioning of the $3 \mathrm{D}$ volume of interest (VOI) and field of view (FOV) of the 3D spectroscopic imaging sequence. This necessitates well-trained technicians with good anatomic knowledge that may not be available in all institutions. The outcome of a prostate MRS exam is therefore highly variable. The complex planning algorithm sometimes paired with lack of expertise puts a high burden on the MR technicians during their daily routine and often yields to prohibitive long examination times.

Because of these limitations and due to the ease of use as well as the proven reliability of functional techniques such as diffusion-weighted imaging (DWI), the PI-RADS guidelines 2.0 recommend spectroscopy only as an optional addendum [15].

However, recent software developments for planning MR examinations by means of an automated user interface promise to improve the feasibility of spectroscopy for clinical routine. Automated scanning protocols facilitate the positioning of scan volumes and saturation bands by recognizing anatomical landmarks in the examination region. Today, the feasibility and effectiveness to achieve shorter and optimized MRI examinations using a variety of automated examination protocols has already been proven in different body regions [16-18], and such software solutions are so far available for routine examinations in the brain, heart, abdomen, and knee. Recently, an experimental solution for the rapidly increasing prostate exams has been developed.

The current study aimed to evaluate the reliability and effectiveness of using this prototype automated user interface protocol for prostate MRS examinations.

\section{Methods \\ Patients}

This prospective study was conducted between November 2012 and February 2014 with the approval of our institutional review boards (Medical University Innsbruck). All participants agreed to participate in the study and provided informed written consent for both participation and publication of the results. Fifty-six patients (age ranged between $44-73$ years, mean 63 years) were included in the study. Of these, 38 patients (age range 4473 years, mean age 61.24 years) were randomly selected to undergo MRS examination with the computer-assisted workflow technique (Prostate DOT Engine, Siemens work-in-progress package 772) and 18 (age range 48-72 years, mean age 61.11 years) underwent conventional manual MRS technique. All patients were referred to our MRI unit from the urology department or the outpatient clinic for MRI examination of the prostate with suspicion of prostate cancer. Patients with general MRI contraindications or claustrophobia as well as local relative contraindications such as recent prostate biopsy were excluded.

\section{mpMRI examination}

All patients underwent mpMRI examination using a $3 \mathrm{~T}$ scanner (MAGNETOM Skyra with software version Syngo MR Numaris 4 VD13A, Siemens Healthcare $\mathrm{GmbH}$, Erlangen, Germany) with a $45 \mathrm{mT} / \mathrm{m}$ gradient system (slew rate $200 \mathrm{~T} / \mathrm{s}$ ). An 18-channel body surface phased-array coil together with a 32-channel spine array coil was used for the examination without placement of an endo-rectal coil. Well-trained technicians with at least 3 years of experience in a university hospital setting performed all exams. 
Multiparametric imaging followed the recommendation of Pi-RADS (v.1) [19] and included a T2-weighted transverse fast spin echo sequence, a transverse spinecho echo planar imaging diffusion-weighted sequence (SE-DWI), MRS, and dynamic contrast-enhanced MRI (DCE MRI). Imaging parameters are summarized in Table 1. For MRS, spin-echo based 3D chemical shift imaging (3D-CSI) [20] was used together with pointresolved volume localization [PRESS]. $(\mathrm{TR} / \mathrm{TE}=940 /$ $145 \mathrm{~ms}$, FOV $99 \mathrm{~mm} \times 90 \mathrm{~mm} \times 90 \mathrm{~mm}$, number of phase encoding steps $11 \times 10 \times 10$, resolution interpolated to $16 \times 16 \times 16$, weighted elliptical scanning with 4 averages, water suppression bandwidth $60 \mathrm{~Hz}$, lipid suppression bandwidth $1.55 \mathrm{ppm}$ at frequency shift of -3.4 ppm, acquisition time $8 \mathrm{~min} 13 \mathrm{sec}$ ). For outer volume suppression [21], 8 saturation regions were placed around the volume of interest with a thickness of $20 \mathrm{~mm}$ each. One saturation region was on-resonance to saturate water signal from the bladder (frequency shift $=0$ $\mathrm{ppm}$ ) while the other saturation regions were used for fat saturation (frequency shift $=-3.4 \mathrm{ppm}$ ).

For the computer-assisted workflow, an automated prototype planning interface was used (DOT engine prototype software, Siemens work-in-progress package 772), which was implemented on the MR scanner by the manufacturer. In this case, the T2w fast spin echo sequence was manually positioned based on initially acquired localizer images. After completion of this sequence, an automatic segmentation process was started during image calculation, aiming to detect the outlines of the prostate. This segmentation process resulted besides the normal $\mathrm{T} 2 \mathrm{w}$ series in an additional image series containing the segmented outlines of the prostate overlaid onto the T2w images, binary images of the segmented prostate, and a report series containing the anatomical extension parameters and total prostate volume. The segmentation algorithm used by the DOT engine prototype is based on three major steps, as described in Birkbeck et al. [22]. First, an intensity normalization procedure is applied on the images for contrast adjustment; then, a statistical shape model based on a 3D deformable mesh derived from a training dataset is aligned to the image data. This means that initial position, orientation, scale, and shape model parameters are estimated based on discriminative classifiers. The final step of the algorithm consists in an iterative refinement of the estimated segmentation by locally deforming the mesh to fit the prostate boundaries.

Based on these segmentation results, the DOT engine automatically positioned all following imaging and spectroscopic series. In particular, the saturation pulses for outer volume suppression were automatically placed in a regular equally distributed fashion around the prostate for the 3D spectroscopic imaging sequence. This is done in a similar way to the approach described by Khurd et al. [23]: the convex hull of the 3D segmentation mesh is computed, and saturation slabs are selected to be tangent to the convex hull along eight spherical directions.

For patients who underwent imaging without the use of the DOT engine planning, the positioning and planning of the imaging, and spectroscopy measurements were performed manually. Scan time and post processing time for MR spectroscopy was identical for patients with and without the use of DOT engine planning.

\section{Post processing}

For all patients, the total examination time was recorded. Spectra were visualized and evaluated using the software provided on the MRI scanner. In all patients, the quality of OVS saturation band placement and spectral quality

Table 1 Imaging parameters for different MRI sequences

\begin{tabular}{llll}
\hline & T2w & DWI & DCE-MRI \\
\hline Sequence type & Fast spin echo & Spin echo EPI & T1w 3D FLASH \\
TR/TE (ms) & $7500 / 101$ & $6800 / 67$ & $4.43 / 1.6$ \\
Echo train length & 25 & 60 & - \\
Slice thickness/gap (mm) & $3.5 / 0$ & $3.5 / 0$ & $3.5 / 0$ \\
FOV ${\text { (mm }{ }^{2} \text { ) }}_{\text {Acquisition matrix }}$ & $200 \times 200$ & $260 \times 208$ & $260 \times 260$ \\
Voxel size mm $^{3}$ & $320 \times 310$ & $160 \times 120$ & $192 \times 140$ \\
Parallel imaging mod (acceleration factor) & $0.6 \times 0.6 \times 3.5$ & $1.6 \times 1.6 \times 3.5$ & $1.4 \times 1.4 \times 3.5$ \\
b values & GRAPPA (2) & GRAPPA (2) & GRAPPA (2) \\
Averages & - & $50,400,1000$ & - \\
Measurements & 2 & $2,3,4$ & 1 \\
Acquisition time (min:sec) & 1 & 1 & 40 \\
\hline
\end{tabular}

T2W T2-weighted sequence, DWI diffusion-weighted imaging, DCE-MRI dynamic Contrast Enhanced Magnetic Resonance Imaging, TR repetition time, TE echo time, 
Table 2 5-point Likert scale evaluation of MRS

\begin{tabular}{|c|c|c|c|}
\hline Rate & Saturation pulse & Spectral quality & Automatic segmentation \\
\hline 1 & $\begin{array}{l}\text { Saturation pulse exactly at the } \\
\text { margin }\end{array}$ & $\begin{array}{l}\text { Spectra all over the margin of the PZ of good } \\
\text { diagnostic quality }\end{array}$ & $\begin{array}{l}\text { Segmentation curve lies exactly at the margin of the } \\
\text { prostate }\end{array}$ \\
\hline 2 & $\begin{array}{l}\text { Gap between the prostate and one } \\
\text { or more of the saturation pulses }\end{array}$ & $\begin{array}{l}\text { Spectra all over the margin of the } \mathrm{PZ} \text { are } \\
\text { minimally distorted but still of good diagnostic } \\
\text { quality }\end{array}$ & $\begin{array}{l}\text { Segmentation curve extends beyond the prostate } \\
\text { margin and covers parts of the peri-prostatic tissues }\end{array}$ \\
\hline 3 & $\begin{array}{l}\text { One or more of the saturation } \\
\text { pulses cover part of the PZ }\end{array}$ & $\begin{array}{l}\text { Spectra all over the margins of the PZ are } \\
\text { markedly distorted with poor diagnostic quality }\end{array}$ & $\begin{array}{l}\text { Segmentation curve covers the prostate, but } \leq 20 \% \\
\text { of the } \mathrm{PZ} \text { are not included }\end{array}$ \\
\hline 4 & $\begin{array}{l}\text { One or more of the saturation } \\
\text { pulses cover the whole PZ }\end{array}$ & $\begin{array}{l}\leq 20 \% \text { of the } \mathrm{PZ} \text { pixels are distorted with poor } \\
\text { diagnostic quality }\end{array}$ & $\begin{array}{l}\text { Segmentation curve covers the prostate, but }>20 \% \\
\text { of the PZ are not included }\end{array}$ \\
\hline 5 & $\begin{array}{l}\text { One or more of the saturation } \\
\text { pulses cover the prostate } \\
\text { completely }\end{array}$ & $\begin{array}{l}>20 \% \text { of the PZ pixels are distorted with poor } \\
\text { diagnostic quality }\end{array}$ & $\begin{array}{l}\text { Segmentation curve totally away from the prostate } \\
\text { (prostate not included within the curve) }\end{array}$ \\
\hline
\end{tabular}

is consensually rated by two experienced radiologists with 7 and 25 years of experience respectively using a 5point Likert scale as summarized in Table 2. In patients where the DOT engine approach was used, we also evaluated the quality of the automatic prostate segmentation comparing the computer-generated outline to the actual prostate margins on the T2-weighted images. All the data were subsequently compared between the two patient groups. As the study aimed to evaluate only the feasibility and impact of the new computer-assisted planning interface, we evaluated 56 consecutive patients that gave written consent irrespective of patient diagnosis, histopathologic analysis, or clinical demographics.

\section{Statistical analysis}

All statistical calculations were performed using the $\mathrm{R}$ Project for Statistical Computing [24]. The obtained total examination times for patients with and without the DOT engine approach were tested for normal distribution using the Shapiro-Wilk normality test and were then compared using either an unpaired Wilcox test (in case of non-normality) or an unpaired $t$ test (in case of normality). For the different Likert scales, frequency tables were calculated and displayed as bar plots. Fisher's exact test, which gives reliable results even for small sample sizes, was used to compare frequencies between patients with and without the DOT engine approach. Results were considered significant for $P$ values below 0.05 .

\section{Results}

\section{Examination time}

The whole examination time for patients, who underwent mpMRI with manual OVS placement for MRS, ranged from 35.45 to 47.25 min (mean 40.78 min, median $40.67 \mathrm{~min}$ ). In contrast, it ranged from 29.58 to $36.42 \mathrm{~min}$ (mean $32.6 \mathrm{~min}$, median $32.58 \mathrm{~min}$ ) for patients who underwent mpMRI with the automated planning approach, the DOT engine. Shapiro-Wilk normality test showed significant normal distribution for automated as well as manual approach with $P=0.824$ and $P=0.752$, respectively. The time reduction observed when using the DOT engine was found to be highly significant $(t$ test, $P<0.001)$.

\section{Saturation pulse positioning}

Quality of saturation pulse band positioning was graded on a scale of 1 to 5 . In our study, all patients except one patient $(17 / 18,94.44 \%)$ were given grade 2 for manual placement of saturation regions, and none were given grade 1 . In comparison, the majority of patients (29 patients out of the total 38 patients or $76.32 \%$ ) examined with the DOT engine MRS were rated as grade 2 and 5 patients (13.16\%) even grade 1. Thus, we observed an optimal placement of the saturation pulses at the prostate margins only in patients with automated MRS. A slight overlap of individual saturation pulses with the prostate gland was detected in $5.6 \%$ of patients with manual approach and $10.5 \%$ of patients with DOT engine MRS.

The rating of the positioning of saturation pulses for OVS is summarized in Table 3. No significant difference was detected between both patient groups $(P=0.227)$.

\section{Spectral quality evaluation}

We also graded spectral quality on a scale of 1 to 5 . For spectral quality, the first three grades $(1,2$, and 3 ) showed nearly the same frequencies in both groups with only mildly better values regarding grade 4 for manual

Table 3 Results of saturation pulse positioning

\begin{tabular}{lll}
\hline Rate & Conventional MRS & Computer-assisted workflow MRS \\
\hline $\mathbf{1}$ & - & $5(13.2 \%)$ \\
$\mathbf{2}$ & $17(94.4 \%)$ & $29(76.3 \%)$ \\
$\mathbf{3}$ & $1(5.6 \%)$ & $4(10.5 \%)$ \\
$\mathbf{4}$ & - & - \\
$\mathbf{5}$ & - & - \\
Total & $18(100 \%)$ & $38(100 \%)$ \\
\hline
\end{tabular}

MRS magnetic resonance spectroscopy 
MRS compared to automated MRS (16.66\% versus $26.32 \%)$. These results, however, have to be considered in perspective with the much better performance of automated MRS compared to manual MRS regarding quality failure (grade 5), which showed a much lower incidence for automated MRS (2.63\%) versus manual MRS (11.11\%). For both methods, the percentage of exams with poor spectral quality (grading 4 and 5) was almost equal at about $28-29 \%$. However, with the automated approach, the occurrence of grade 5 exams was clearly reduced.

Spectral quality rating for both patient groups is summarized in Table 4. Statistically again, no significant difference was detected between both groups $(P=0.598)$.

\section{Prostate segmentation quality}

Prostate segmentation could only be evaluated in patients who underwent mpMRI with DOT engine approach. Inline segmentation showed good performance with most of the patients being rated as grades 1 and 2 (25 out of $38,65.79 \%$ ) while in 13 patients $(34.21 \%)<$ $20 \%$ of the PZ was clipped by the automatic segmentation curve. More than 20\% clipping or total segmentation failure was not observed at all.

The results of automatic segmentation are summarized in Table 5.

\section{Discussion}

MRS has the potential to differentiate between benign and malignant lesions of the prostate based on metabolite changes. However, it faces a lot of challenges that hamper its use in routine clinical work such as the need for exact coil positioning and meticulous acquisition planning with precise saturation pulse placement thus leading to a long examination time $[6,13]$.

In the past years, scanner manufacturers have begun to develop new automated image acquisition techniques aiming at facilitating, optimizing, and standardizing patient examinations. In case of MR exams, even complex protocols can be conducted reliably irrespective of the individual operator's experience. Recently, an experimental automated scanning protocol was developed for prostate mpMRI. In our study, we evaluated the

Table 4 Results of spectral quality rating

\begin{tabular}{lll}
\hline Rate & Conventional MRS & Computer-assisted workflow MRS \\
\hline $\mathbf{1}$ & $6(33.3 \%)$ & $14(36.8 \%)$ \\
$\mathbf{2}$ & $2(11.1 \%)$ & $2(5.3 \%)$ \\
$\mathbf{3}$ & $5(27.8 \%)$ & $11(28.9 \%)$ \\
$\mathbf{4}$ & $3(16.7 \%)$ & $10(26.3 \%)$ \\
$\mathbf{5}$ & $2(11.1 \%)$ & $1(2.6 \%)$ \\
Total & $18(100 \%)$ & $38(100 \%)$ \\
\hline
\end{tabular}

MRS magnetic resonance spectroscopy
Table $\mathbf{5}$ Results of automatic prostate segmentation by DOT engine MRS

\begin{tabular}{ll}
\hline Rate & Computer-assisted workflow MRS \\
\hline $\mathbf{1}$ & $24(63.2 \%)$ \\
$\mathbf{2}$ & $1(2.6 \%)$ \\
$\mathbf{3}$ & $13(34.2 \%)$ \\
$\mathbf{4}$ & - \\
$\mathbf{5}$ & - \\
Total & $38(100 \%)$ \\
\hline
\end{tabular}

MRS magnetic resonance spectroscopy

scanning efficacy of such an automated workflow versus conventional mpMRI scanning technique.

In our patient group with conventional mpMRI, the total scanning time was around $40.78 \mathrm{~min}$, which was slightly shorter than indicated in literature. In a previous study by Yamamura et al. [14], a total scanning time of about $41 \mathrm{~min}$ was reported for prostate imaging including MRS albeit omitting DCE MRI. However, Fusco et al. [12] showed a longer scanning duration of about 50 min for a similar scanning protocol to ours that included DCE MRI. In contrary to these studies conducted on $1.5 \mathrm{~T}$ scanners, we used a $3 \mathrm{~T}$ MRI scanner with surface coil placement allowing for somewhat shorter scanning times.

In our second group of our patients with automated scanning strategy using the DOT engine approach, we achieved a significant reduction of the whole examination time (mean difference $8 \mathrm{~min}$ ) compared to the non-automated patient group $(P<0.001)$. Our mean total examination time was actually shorter than the total scan time given in literature by Wetter et al. who used a mpMRI protocol without DWI and DCE MRI [6]. To the best of our knowledge, no prior studies have evaluated the role of a guided and automated workflow such as the DOT engine prototype for prostate MRI examinations. Moenninghof et al. [18] evaluated the role of an automated protocol for brain imaging and its impact on head examinations using $1.5 \mathrm{~T}$ MRI scanners. They showed a significant reduction of the total examination time that decreased from 24:53 to 20:01 min when using the automated approach as well as a significant reduction of the radiology technician activity time $(P<$ $0.001)$. Our results in the prostate are similar to theirs proving equally significant time reduction $(P<0.001)$.

On modern MR scanners, planning of consecutive scans is performed during the acquisition of previous sequences. In case of prostate spectroscopy, the planning task is very complex and takes considerable longer time than the available time span of prior scans even for highly trained technicians (Figs. 1 and 2). Thus, a "thinking" pause is prone to occur between two scans prolonging the overall examination time. Our results prove that 

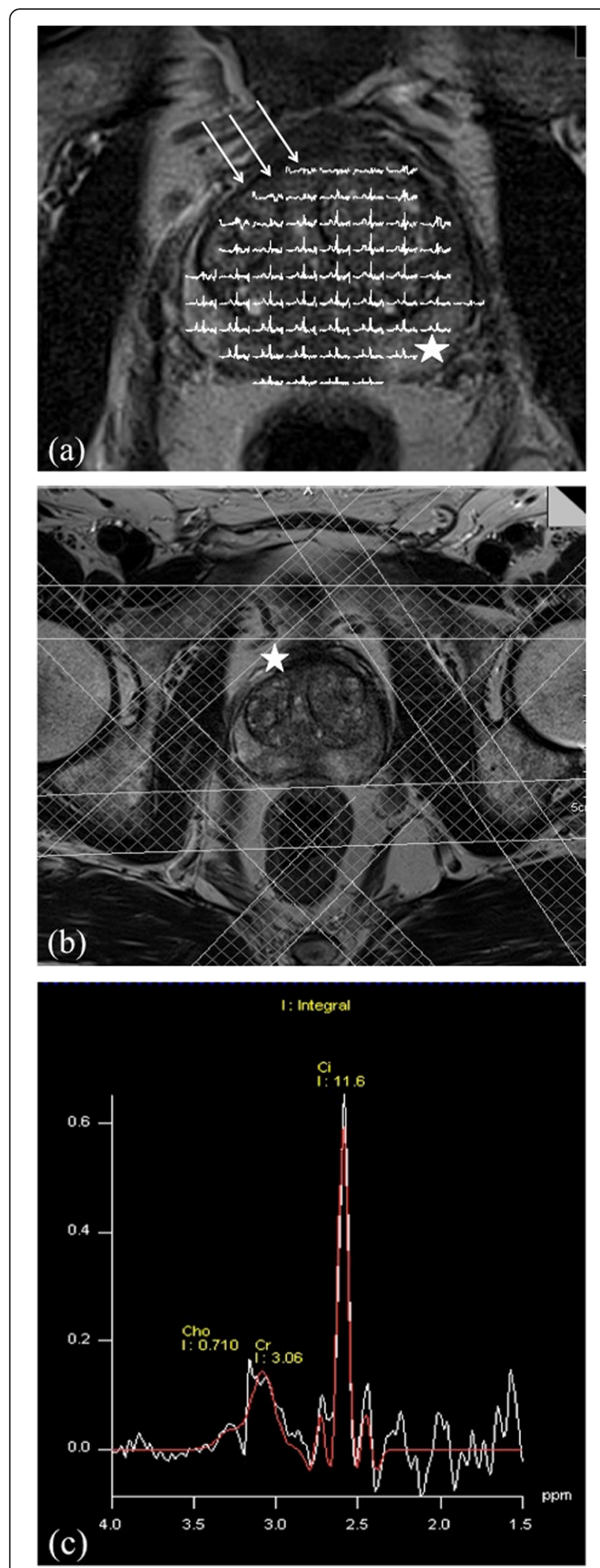

Fig. 1 Prostate spectroscopy with conventional planning of saturation pulses, acquisition matrix, and field of view. Small posterolateral parts of the prostatic gland were not included (a white star) and low quality of several spectroscopic voxels in the right anterior region of the prostatic gland (a: white arrows). This may be due to imprecise positioning of saturation pulses (b, white star: Saturation pulse is not exactly aligned to the margin of the prostatic gland). Sample spectrum of the mid-peripheral zone showing acceptable diagnostic quality (c)

this time delay is omitted using an automated planning algorithm allowing an examination time reduction of about $20 \%$. On the other hand, it has been shown that a computer-assisted interface in a straightforward knee examination does not provide any significant time reduction [25]. However, the authors still assume that the avoidance of operator errors and even a minimal nonsignificant time saving per exam would make a financial return by the end of the day likely to occur.

The quality of the spectra is highly dependent on the precise placement of the numerous saturation pulses around the outer circumference of the prostate. We could show that the automated approach of saturation band placement with the DOT engine outperformed the manual positioning of the background suppression pulses. Optimal placement of the saturation bands and thus ideal background suppression was achieved only in the patient group with the automated approach. On the other hand, a slightly more frequent clipping of the prostate surface occurred in the DOT engine group but did not significantly degrade spectral quality according to our scale. We could not find any significant differences in the occurrence of spectra rated 1 to 4; however, the number of unacceptable quality spectra (grade 5) was much lower in the automated group compared to the manual group (2.63\% versus $11.11 \%)$.

The primary algorithm of prostate segmentation by the DOT engine still needs some improvement. In about one third of patients (13 out of 38), surface clipping of the PZ occurred. In these patients, the total prostate volume is underestimated yielding to an erroneous calculation of an important tumor marker, the PSA density, which is the relationship between the measured PSA level in the serum and the prostate volume. Most importantly partial shading of MRS voxels by saturation bands will most probably lead to a decreased sensitivity to metabolic changes. This however is highly undesirable in these surface areas of the prostate since about $75 \%$ of all prostate cancers originate in the outer zone of the gland.

Although we could clearly demonstrate the time saving benefit of the automated scanning protocol, our study has some limitations.

First, the sample size in our study was relatively low so that our results have to be treated with caution, and 

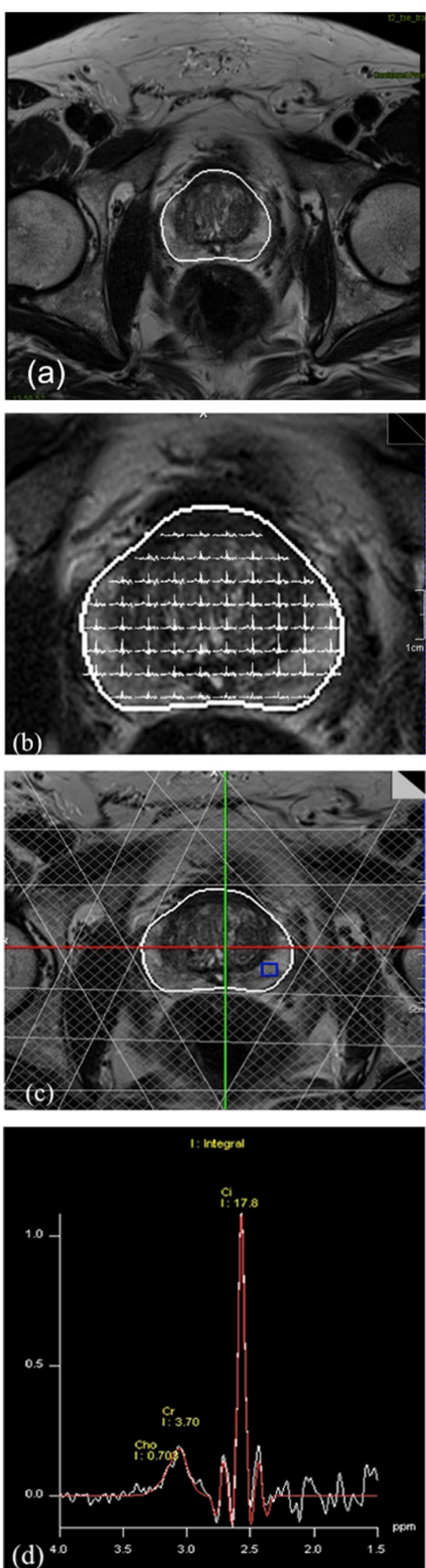

Fig. 2 Prostate spectroscopy with automatic DOT engine prototype. Automatic planning of saturation pulses, acquisition matrix, and field of view. Note precise delineation of the prostatic gland, which is seen within the automatic segmentation curve. a Axial image of the prostate gland with the surrounding white automatic segmentation curve precisely located at the margin of the prostate. $\mathbf{b}$ Axial image of the prostate gland showing all spectroscopic voxels inside the area delineated by the automatic segmentation curve. c Axial image of the prostate with saturation pulses around the prostate, which are accurately located at prostatic margins. $\mathbf{d}$ Sample spectrum of the mid-peripheral zone showing good diagnostic quality

further studies would be advisable. Second, we only examined the feasibility of the technique and the resulting image quality by means of a consensual evaluation by two experienced radiologists with no inter-observer correlation. In a clinical setting, however, the specificity and sensitivity of the acquired measurements need to be compared.

\section{Conclusion}

Our results show that the assisted workflow for prostate MRS achieved good spectral quality in a significantly reduced examination time when compared to conventional MRS. Its ease of use and higher workflow efficiency may facilitate the inclusion of MRS in clinical routine.

\section{Abbreviations \\ MRS: Magnetic resonance spectroscopy; MRI: Magnetic resonance imaging; mpMRI: Multiparametric MRI; DWI: Diffusion-weighted imaging; DCE MRI: Dynamic contrast-enhanced MRI; OVS: Outer volume suppression; DOT: Day optimizing throughput; SE-DWI: Spin-echo echo planar imaging diffusion-weighted sequence; 3D-CSI: 3D chemical shift imaging}

\section{Acknowledgements}

No

\section{Authors' contributions}

All authors shared in the preparation and construction of the frame of the manuscript. MAE, CK, WJ, and DJ shared in performing the examinations and analyzing the data. BK, MR, and RN provided us with the software and training how to use it, but they did not share in data collection, analysis, or interpretation. All authors again shared in writing and editing the manuscript and its final approval.

Funding

No funding.

\section{Availability of data and materials}

The datasets used and/or analyzed during the current study are available

from the corresponding author on reasonable request.

\section{Ethics approval and consent to participate}

The medical university of Innsbruck (MUI) gave ethics approval.

The reference number of the ethics committee is AN4819 316/4.21

A written informed consent was obtained from all study participants.

\section{Consent for publication}

Not applicable

Competing interests

All authors declare no conflict of interest. 


\section{Author details}

${ }^{1}$ Department of Radiology, Medical University Innsbruck, Anich St. 35, 6020 Innsbruck, Austria. ${ }^{2}$ Department of Diagnostic Radiology, South Egypt Cancer Institute, Assiut University, Assiut, Egypt. ${ }^{3}$ Department of Radiology / Neuroradiology Rehhabilitation Clinic UIm (RKU), Ulm, Germany. ${ }^{4}$ Siemens Healthcare GmbH, Erlangen, Germany. ${ }^{5}$ Siemens Healthcare Limited, Firmley, UK.

Received: 21 November 2019 Accepted: 17 March 2020

Published online: 06 April 2020

\section{References}

1. Vilanova JC, Barcelo J (2007) Prostate cancer detection: magnetic resonance [MR] spectroscopic imaging. Abdom Imaging. 32(2):253-261

2. Levi F, Lucchini F, Negri E, Boyle P, La Vecchia C (2004) Leveling of prostate cancer mortality in Western Europe. Prostate. 60(1):46-52

3. Hayat MJ, Howlader N, Reichman ME, Edwards BK (2007) Cancer statistics, trends, and multiple primary cancer analyses from the Surveillance, Epidemiology, and End Results [SEER] Program. Oncologist. 12(1):20-37

4. Bray F, Ferlay J, Soerjomataram I, Siegel RL, Torre LA, Jemal A (2018) Globa cancer statistics 2018: GLOBOCAN estimates of incidence and mortality worldwide for 36 cancers in 185 countries. CA Cancer. J Clin 68:394-424

5. Chen H, Sutedjo J, Wang L, Yin X (2016) Prostate cancer magnetic resonance spectroscopy imaging at 1.5 and $3.0 \mathrm{~T}$ : a meta-analysis. Technol Cancer Res Treat. 15(4):625-631

6. Wetter A, Hubner F, Lehnert T et al (2005) Three-dimensional 1H-magnetic resonance spectroscopy of the prostate in clinical practice: technique and results in patients with elevated prostate-specific antigen and negative or no previous prostate biopsies. Eur Radiol. 15(4):645-652

7. Weis J, von Below C, Tolf A et al (2017) Quantification of metabolite concentrations in benign and malignant prostate tissues using 3D proton MR spectroscopic imaging. J Magn Reson Imaging. 45(4):1232-1240

8. Villeirs GM, De Meerleer GO, De Visschere PJ, Fonteyne VH, Verbaeys AC, Oosterlinck W (2011) Combined magnetic resonance imaging and spectroscopy in the assessment of high grade prostate carcinoma in patients with elevated PSA: a single-institution experience of 356 patients. Eur J Radiol. 77(2):340-345

9. Porzycki P, Ciszkowics E (2019) Detection of individual prostate cancer via multiparametric magnetic resonance imaging in own material - initial experience. J Contemp Brachitherapy. 11(6):541-546

10. Wroclawski ML, Kayano PP, Amaral BS et al (2020) Can multiparametric magnetic resonance of the prostate avoid biopsies in patients with elevated PSA and surgical indication for benign prostatic enlargement? Abdom Radiol (NY). https://doi.org/10.1007/s00261-020-02411-y

11. Choi YJ, Kim JK, Kim N, Kim KW, Choi EK, Cho KS (2007) Functional MR imaging of prostate cancer. Radiographics 27(1):63-75 discussion -7

12. Fusco R, Sansone M, Petrillo M et al (2016) Multiparametric MRI for prostate cancer detection: preliminary results on quantitative analysis of dynamic contrast enhanced imaging, diffusion-weighted imaging and spectroscopy imaging. Magn Reson Imaging. 34(7):839-845

13. Kim JK, Jang YJ, Cho G (2009) Multidisciplinary functional MR imaging for prostate cancer. Korean J Radiol. 10(6):535-551

14. Yamamura J, Salomon G, Buchert R et al (2011) MR imaging of prostate cancer: diffusion weighted imaging and [3D] hydrogen $1[\mathrm{H}] \mathrm{MR}$ spectroscopy in comparison with histology. Radiol Res Practice. 2011: 616852. https://doi.org/10.1155/2011/616852 PubMed PMID: 22091382; PubMed Central PMCID: PMC3198606

15. Muller BG, Shih JH, Sankineni S et al (2015) Prostate cancer: interobserver agreement and accuracy with the revised prostate imaging reporting and data system at multiparametric MR imaging. Radiology. 277(3):741-750

16. Lu X, Jolly MP, Georgescu B et al (2011) Automatic view planning for cardiac MRI acquisition. Med Image Comput Comput Assist Interv 14(Pt 3): 479-486 PubMed PMID: 22003734

17. Bauer S, Ritacco LE, Boesch C, Nolte LP, Reyes M (2012) Automatic scan planning for magnetic resonance imaging of the knee joint. Ann Biomed Eng. 40(9):2033-2042

18. Moenninghoff C, Umutlu L, Kloeters C et al (2013) Workflow efficiency of two 1.5 T MR scanners with and without an automated user interface for head examinations. Acad Radiol 20(6):721-730
19. Barentsz JO, Richenberg J, Clements R, Choyke P, Verma S, Villeirs G et a (April 2012) ESUR prostate MR guidelines 2012. Eur Radiol 22(4):746-757. https://doi.org/10.1007/s00330-011-2377-y

20. Brown TR, Kincaid BM, Ugurbil K (1982) NMR chemical shift imaging in three dimensions. Proc Natl Acad Sci U S A 79(11):3523-3526 PubMed PMID: 6954498; PubMed Central PMCID: PMC346453

21. Tran TK, Vigneron DB, Sailasuta N et al (2000) Very selective suppression pulses for clinical MRSI studies of brain and prostate cancer. Magn Reson Med 43(1):23-33

22. Birkbeck N, Zhang J, Requardt M, Kiefer B, Gall P, Kevin ZS (2012) Regionspecific hierarchical segmentation of MR prostate using discriminative learning. MICCAI Grand Challenge: Prostate MR Image Segmentation, p 2012

23. Khurd P, Grady L, Gajera K et al (eds) (2011) Facilitating 3d spectroscopic imaging through automatic prostate localization in mr images using random walker segmentation initialized via boosted classifiers. Springer

24. Team RDC (2011) R: a language and environment for statistical computing. R Foundation for Statistical Computing

25. Shawn Kisch PB, Plewes C, Vanderby S (2015) Effect of day optimizing throughput [DOT] knee software implementation on magnetic resonance imaging workflow efficiency. J Med Imaging Radiat Sci 46(4):420-426

\section{Publisher's Note}

Springer Nature remains neutral with regard to jurisdictional claims in published maps and institutional affiliations.

\section{Submit your manuscript to a SpringerOpen ${ }^{\circ}$ journal and benefit from:}

- Convenient online submission

- Rigorous peer review

- Open access: articles freely available online

- High visibility within the field

- Retaining the copyright to your article

Submit your next manuscript at $>$ springeropen.com 